\title{
The curious case of vacuolar ATPase: regulation of signaling pathways
}

\author{
Sahithi Pamarthy ${ }^{1,2}$, Arpita Kulshrestha', Gajendra K. Katara ${ }^{1}$ and Kenneth D. Beaman ${ }^{1 *}$
}

\begin{abstract}
The Vacuolar ATPase (V-ATPase) is a proton pump responsible for controlling the intracellular and extracellular pH of cells. The structure of V-ATPase has been highly conserved among all eukaryotic cells and is involved in diverse functions across species. V-ATPase is best known for its acidification of endosomes and lysosomes and is also important for luminal acidification of specialized cells. Several reports have suggested the involvement of V-ATPase in maintaining an alkaline intracellular and acidic extracellular pH thereby aiding in proliferation and metastasis of cancer cells respectively. Increased expression of V-ATPase and relocation to the plasma membrane aids in cancer modulates key tumorigenic cell processes like autophagy, Warburg effect, immunomoduation, drug resistance and most importantly cancer cell signaling. In this review, we discuss the direct role of V-ATPase in acidification and indirect regulation of signaling pathways, particularly Notch Signaling.
\end{abstract}

Keywords: V-ATPase, Cancer, mTOR, WNT, TGF- $\beta$, Notch signaling, Autophagy, Drug resistance, Warburg effect

\section{Background}

The Vacuolar ATPase (V-ATPase) is a multi-subunit ATP driven proton pump that acidifies intracellular vesicles and extracellular milieu and thereby is involved in a large number of biological functions [1]. Previous reviews have elegantly described the structure and function of V-ATPase [2-4]. Here we review the recent literature pertaining to VATPase function and contribution to various cell processes in normal physiology with an emphasis on cancer. We also present in detail the regulation of Notch and other signaling pathways by V-ATPase revealing a hitherto less known function of V-ATPase in cell signaling.

\section{Subunit isoforms}

Structurally, the V-ATPase is a rotary nanomotor made up of multiple subunits, each with multiple isoforms [5]. Subunits are arranged in two domains: a peripheral $V_{1}$ domain, responsible for ATP hydrolysis and an integral membrane domain $V_{O}$, which functions in proton translocation. The structure of V-ATPase has been highly conserved among all eukaryotic cells and is involved in diverse functions across species. In mammals, $\mathrm{V}_{1}$ domain has eight different subunits $(A, B, C, D, E, F, G$ and $H)$ whereas the $\mathrm{V}_{\mathrm{O}}$ domain is composed of six different subunits $\left(a, c, c, c^{\prime \prime}, d, e\right)$ [6]. The differential requirement of acidification in intracellular vesicles and extracellular milieu drives V-ATPase function and regulation. To decrease or increase pump efficiency VATPase controls the coupling between ATP hydrolysis and proton pumping. This process is brought about by the ' $a$ ' subunit of V-ATPase [7]. Similarly, cell and compartment specific targeting of V-ATPase is also dependent on the 'a' subunit isoforms. $\mathrm{V}_{\mathrm{O}} a$ is a $100-\mathrm{kDa}$ integral membrane protein with an $\mathrm{N}$ terminal cytosolic tail and 9 transmembrane domains. Four isoforms of the ' $a$ ' subunit ( $a 1, a 2$, a3 and a4) have been identified with distinct vesicular and cell type distribution. $V_{\mathrm{O}} a 1$ is expressed on the synaptic vesicles and $\mathrm{V}_{\mathrm{O}} a 2$ is expressed on intracellular vesicles like golgi and early endosomes. $\mathrm{V}_{\mathrm{O}} a 3$ is expressed on plasma membrane of osteoclasts, whereas $\mathrm{V}_{\mathrm{O}} a 4$ is expressed on the plasma membrane of renal intercalated cells. Further, the $\mathrm{N}$-terminus of subunit ' $\mathrm{a}$ ' is an important motif that tethers V1 domain to the membrane and has also been reported to be a unique $\mathrm{pH}$ sensor in lysosomes [8]. The expression and isoform localization of subunit ' $a$ ' is critical to the functioning of V-ATPase [5].

\footnotetext{
* Correspondence: kenneth.beaman@rosalindfranklin.edu

${ }^{1}$ Department of Microbiology and Immunology, Rosalind Franklin University of

Medicine and Science, 3333 Green Bay Road, North Chicago, IL 60064, USA

Full list of author information is available at the end of the article
} 


\section{Physiological function of V-ATPase}

The V-ATPase is ubiquitously expressed and performs diverse biological functions within cells of most tissues through vesicular, luminal and extracellular acidification [9]. To achieve numerous cellular functions, V-ATPase facilitates localized concentration of protons in acidic vesicles of the endocytic and exocytic pathways [1].

\section{Vesicular acidification}

\section{Endosomes and lysosomes}

V-ATPase is best known for its role in the acidification of intracellular vesicles like endosomes and lysosomes. On the surface of endosomes, V-ATPase acidifies and thereby modulates important cellular processes like receptor endocytosis and vesicular trafficking [10]. Acidification of endosomes by V-ATPase is crucial for endocytic internalization of receptor ligand complexes. Following signaling initiation, lower $\mathrm{pH}$ in endosomes releases the ligand recycling it to the plasma membrane [11]. In lysosomes, V-ATPases help maintain the low $\mathrm{pH}$ of 4.5 and are also important for transportation of newly synthesized acid hydrolases from Golgi to lysosomes. Further, phagosomes and autophagosomes in macrophages and tumor cells respectively, also depend on the acidic $\mathrm{pH}$ maintained by V-ATPase for the activity of the degradative enzymes in these compartments [12].

\section{Golgi}

The sorting of exocytic and endocytic machinery begins at the golgi complex. Importantly, most proteins undergo glycosylation, a crucial posttranslational modification within the golgi apparatus [13]. Mutations in the a2 subunit of VATPase result in cutis laxa, an autosomal recessive wrinkly skin syndrome wherein impaired glycosylation of extra cellular matrix proteins is observed [14]. Although V-ATPase has been genetically correlated to glycosylation defect, the exact relationship between golgi acidification and protein maturation has not been explored.

\section{Specialized vesicles}

$\mathrm{V}$-ATPase is a major protein expressed in specialized compartments of specific cell types. During neurotransmission, V-ATPase provides the crucial proton motive force necessary for the formation of synaptic vesicles and subsequent accumulation of neurotransmitters [15]. In pancreatic cells, V-ATPase dependent acidification is important for insulin exocytosis [16]. V-ATPase also governs the fission-fusion balance of vesicular system by interacting with Soluble NSF Attachment protein Receptor (SNARE) and GTPase [17].

\section{Luminal acidification}

V-ATPases were initially identified on intracellular vesicles, but the importance of plasma membrane V-ATPases has grown enormously [18]. In the epithelial cells of proximal tubule of kidney, the a4 isoform of V-ATPase maintains acid base balance and acidification of urine (systemic acidosis) $[19,20]$. Similarly, in clear cells of the epididymis, plasma membrane V-ATPase acidifies the luminal compartment and helps in sperm maturation and storage [21, 22]. In osteoclasts of the bone, lysosomal V-ATPase translocates to the plasma membrane during bone resorption to acidify the lacunae [23]. Plasmalemmal V-ATPase is crucial to the functioning of interdental cells of the ear, epithelial cells of the nose and vision [24-26]. V-ATPase dysfunction is associated with pathological conditions like renal tubular acidosis, deafness, impairment of olfactory sense, and osteoporosis [27-29] A schematic outlining the role of V-ATPase in vesicular and luminal acidification is shown in Fig. 1.

\section{Role in cancers}

Recently, plasma membrane V-ATPase has been extensively studied in cancer, where they help maintain an alkaline intracellular environment favorable for growth and an acidic extracellular environment favorable for invasion [30]. In tumors, V-ATPase expression has been shown to be higher towards the leading edge of proliferating cancer cells of breast, prostate, lung, ovarian, liver, pancreatic, melanoma and esophageal cancers [2]. Specifically, breast cancer cells express V-ATPase on the plasma membrane to acidify extracellular space and the quantitative expression of V-ATPase correlates with invasiveness and metastatic potential of the cell line [31]. The exact contribution of V-ATPase to the growing tumor is achieved through its influence on the molecular mechanisms/pathways discussed below.

\section{Immunomodulation}

The $a 2$ isoform of Vacuolar ATPase $\left(\mathrm{V}_{\mathrm{O}} a 2\right.$ or $\left.a 2 \mathrm{~V}\right)$ has an immunomodulatory role in pregnancy and cancer. Studies involving $a 2 \mathrm{~V}$ in reproductive biology unearthed a hitherto unknown role for this molecule in normal sperm maturation and production in addition to embryo implantation [22, 32]. In the tumor microenvironment, the $\mathrm{N}$ terminal domain of a2 $\mathrm{V}$ polarizes macrophages to the tumor-associated macrophages (M2 type) and stimulates different monocyte subsets through endocytosis pathway [33]. Following these findings, it was further demonstrated that $a 2 \mathrm{~V}$ deficiency in tumor cells alters the resident macrophage population in the tumor microenvironment and affects in vivo tumor growth [34]. $a 2 \mathrm{~V}$ is expressed on the primary granules of neutrophils and helps maintain $\mathrm{pH}$ in exocytic pathway during neutrophil activation [35]. Treatment of human neutrophils with recombinant $\mathrm{N}$ terminal peptide of a2V (a2NTD) promoted neutrophil migration and polarization [36]. Together, these studies highlight the immunomodulatory role of V-ATPase in eliciting potent immune responses.

\section{Warburg effect}

A hallmark of cancer is the Warburg effect where cells shift from oxidative phosphorylation to aerobic glycolysis 


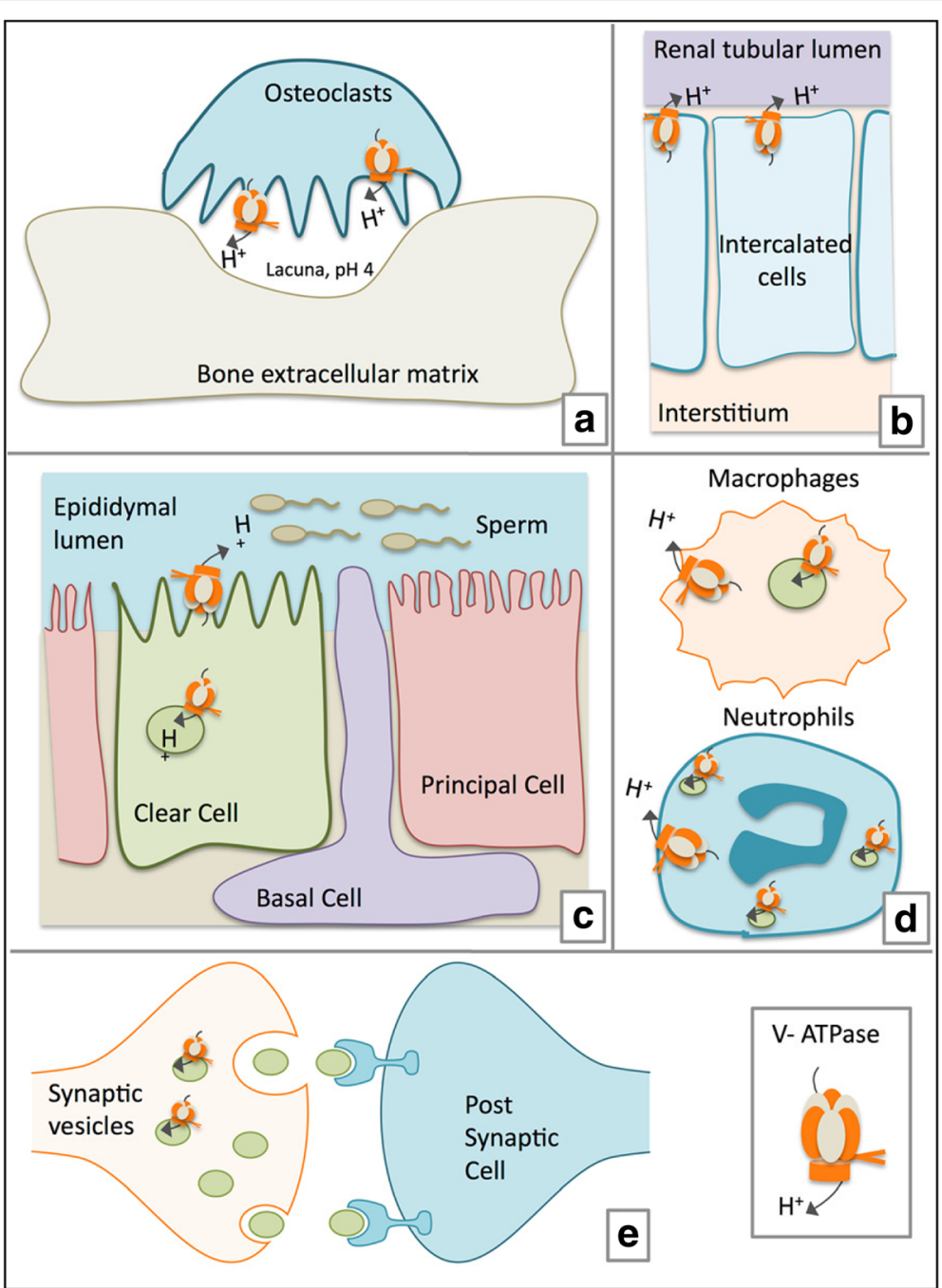

Fig. 1 Physiological role of V-ATPase in luminal acidification. The involvement of V-ATPase is involved in numerous specialized cell processes including bone resorption, renal function, sperm maturation, innate immune responses and neurotransmission is outlined. a Bone resorption: V-ATPase located on the plasma membrane of osteoclasts mediates extracellular acidification for bone demineralization during bone resorption. b Renal function: In the kidney, intercalated cells maintain systemic acidosis and achieve urinary acidification by proton pumping activity of V-ATPases expressed on apical membrane. c Sperm maturation: In the epididymis, V-ATPase expressing clear cells acidify the lumen, a process that is crucial for the proper maturation and motility of spermatozoa. $\mathbf{d}$ Innate immune responses: V-ATPases mediated vesicular acidification has an important role in trafficking and exocytosis of neutrophil granules V-ATPase is constitutively expressed on the plasma membrane of monocytes and activated lymphocytes and contributes to $\mathrm{pH}$ related inflammatory responses. e Neurotransmission: V-ATPase provides the crucial proton motive force necessary for the formation of synaptic vesicles and subsequent accumulation of neurotransmitters. V-ATPase provides the crucial electrochemical potential necessary for accumulation of neurotransmitters in the secretory synaptic vesicles

[37]. Several studies point to the hypothesis that cancer cells depend on V-ATPase more than any other $\mathrm{pH}$ regulators like $\mathrm{Na}^{+} \mathrm{H}^{+}$exchangers, bicarbonate transporters and proton-lactate symporters to achieve the favorable alkaline intracellular $\mathrm{pH}$ and acidic extracellular $\mathrm{pH}$ [38]. Alkalization of cytosol activates glycolysis while suppressing oxidative phosphorylation [39]. Further, some glycolysis related oncogenes like Hypoxia Induced Factor (HIF-1) are regulated by $\mathrm{pH}$ alteration induced by V-ATPase [40].

\section{Acid proteases}

Consequent to extracellular acidification in tumors is the activation of acid proteinases, which are enzymes that cleave the extracellular matrix during tumor invasion. These enzymes belong to the class of acid proteinases like cathepsins [41], Matrix Metallo Proteinases (MMP) and gelatinases and are active at acidic $\mathrm{pH}[42,43]$. Furthermore, the activity of intracellular enzymes like $\gamma$-secretase, which are active at acidic $\mathrm{pH}$, is also enhanced with increased activity of V-ATPase in vesicles [44]. Subsequently, 
this leads to dysregulation of oncogenic pathways like Notch.

\section{Drug resistance and V-ATPase inhibitors}

Altered $\mathrm{pH}$ of tumor microenvironment may influence sensitivity to chemotherapeuticdrugs [45]. Anthracyclines and alkaloids have a pKa of 7 to 8 and are internalized to the endosomal compartment [46]. Recent data suggests that use of V-ATPase inhibitors not only causes cytosolic $\mathrm{pH}$ alterations leading to cell death but also enhances drug uptake, thereby making an effective component of combinatorial treatment to cancer [47]. In ovarian cancer, $a 2 \mathrm{~V}$ is expressed on the leading edge of cancer cells and modulates the activity of MMP9. Further, a2V contributes in cisplatin mediated drug resistance in ovarian cancer and selective inhibition of a2V could serve as an efficient strategy to treat chemoresistant ovarian cancer [48]. The V-ATPase inhibitors bafilomycin and concanamycin belong to a class of pleomacrolides that target the $\mathrm{V}_{\mathrm{O}}$ sector and efficiently inhibit V-ATPase activity. Recently, Apicularen and archazolids have been reported to be potent and specific inhibitors of V-ATPase [49]. However, all available small molecule inhibitors have significant toxicity considering the involvement of V-ATPase in normal cell physiology [50]. Therefore development of specific neutralizing antibodies against the ' $a$ ' subunit isoform that has cell specific expression could be an efficient alternative to cause direct V-ATPase inhibition while also tackling multi drug resistance indirectly with combinatorial use [51].

\section{Autophagy}

Autophagy is the process of selective degradation or recycling of cargos delivered by autophagosomes to lysosomes [52]. Tumor cells show varied dependence on autophagy as they progress from primary tumor to the highly metastatic solid tumor [53]. Cellular cargo marked for degradation are delivered to the lysosomes by autophagic processes. The proton pumping activity of V-ATPase is responsible for activation of lysosomal acid hydrolases which degrade cargo uptake from autophagosomes [54]. Although studies point to the requirement of functional V-ATPase for autophagy [55] and V-ATPase inhibitor Bafilomycin is used as classic inhibitor of autophagy [56], the exact role of V-ATPase in membrane dynamics of autophagic flux is not understood. A recent study reported that treatment with Bafilomycin, which inhibits the activity of both V-ATPase and $\mathrm{Ca}^{2+}$ pump SERCA pump led to blockade in autophagic flux whereas V-ATPase deficient lysosomes were still capable of fusing with autophagosomes [57]. These results suggest the involvement of V_ATPase in degrading autophagic cargo in lysososomes than in autophagic flux and highlight the need for developing specific inhibitors and gene manipulation techniques to study the exact role of V-ATPase in various important cell processes.

\section{Signaling}

The endolysosomal pathway is important for both positive and negative regulation of signaling pathways $[8,58]$. The first known report of involvement of V-ATPase in signaling came from a study showing that inhibition of V-ATPase by Bafilomycin affected internalization of EGFR [59]. Since then, V-ATPase has been associated with signal transduction [60] associated with m-TOR (mammalian Target Of Rapamycin), Wnt, TGF- $\beta$ and Notch Signaling regulation.

\section{Notch signaling}

Perhaps the most well studied signaling pathways regulated by V-ATPase is Notch. This can be attributed to the fact that Notch signaling depends on the endolysosomal pathway for its activation, maintenance and degradation of key pathway mediators [61-63]. V-ATPase maintains cellular $\mathrm{pH}$ balance and plays an important role in endocytosis, protease activation and protein degradation. Specifically, a2V (VATPase subunit- $\mathrm{V}_{\mathrm{O}}$ a2) was previously localized to early endosomes - the site for receptor endocytosis [8]. Following ligand binding, Notch receptor takes the endocytic route and is cleaved by proteases for activation. Later, the receptors are degraded in the lysosome [63]. In Drososphila, mutations in Vps25, a component of ESCRT machinery that regulates endosomal sorting of signaling receptors, causes accumulation of the Notch receptor in endosomes and enhances Notch signaling [64]. In a study analyzing drosophila mutations of Hrs, another component of ESCRT, Notch accumulates in endosomes but does not cause ectopic activation of Notch signaling [65]. The loss of autophagy leads to activation of the Notch signaling in the Drosophila ovarian follicle cells due to disruption of Notch degradation [66]. Contrary to these reports, an independent study found that mutations in Rabconnection-3 disrupt the proton-pumping activity of V-ATPase and accumulate Notch in late endosomes after S2 cleavage, thereby reducing Notch Signaling in Drosophila and mammalian cells [67]. These findings were followed by reports in Drosophila further indicating that through the acidification of endolysosomal pathway, VATPase is required for the activation of Notch in endosomes as well as for the degradation of Notch in lysosomes [68]. During mammalian development, expression of a dominant negative subunit of V-ATPase in neural precursors reduced Notch signaling and depleted neural stem cells leading to neuronal differentiation [69]. Recently, studies in astrocytes in the retina of Nuc1 mutated rats were shown to dysregulate Notch signaling. The reduction in Notch signaling was due to mutated $\beta A 3 / A 1-c r y s t a l l i n$, which regulates VATPase activity resulting in impaired endosomal acidification and $\gamma$-secretase activity thereby affecting the rate of Notch receptor processing [70]. This is an interesting finding considering that the role of V-ATPase in vision in now emerging [26]. Together these findings indicate that the regulation of Notch signaling by V-ATPase can have both 
positive and negative outcomes depending on the cellular localization of V-ATPase activity affected (endosomes vs lysosomes) and the dependence of Notch receptor processing on the endosomal pathway [71, 72]. Although the V-ATPase and Notch crosstalk has been investigated in the context of V-ATPase dependent endolysosomal acidification affecting Notch signaling, a recent report suggests that regulation could also be vice-versa. Specifically, the authors suggest that Presinilin1 (PS1), a component of the $\gamma$-secretase enzyme complex responsible for cleavage of Notch receptor and $\beta$-amyloid peptide physically interacts with the $\mathrm{V}_{\mathrm{O}} a 1$ isoform of V-ATPase and targets it from the endoplasmic reticulum to the lysosomes [73]. Our studies have identified that V-ATPase regulates Notch Signaling in breast cancer [74] and mammary gland development [75]. a2V is expressed on the surface of proliferating mammary epithelial cells and Triple Negative Breast Cancer (TNBC) cells, indicating its role in cell proliferation during normal development and disease. In TNBC, a2V inhibition enhances Notch Signaling by blocking lysosomal and autophagic degradation of Notch receptor [74]. Loss of $a 2 \mathrm{~V}$ in mouse mammary gland leads to abnormal Notch activation and impairs ductal morphogenesis, causing lactation defects [75]. Notch signaling is activated during preterm labor induced by infection with $\mathrm{PGN}+$ poly (I:C), resulting in upregulation of proinflammatory responses, and its inhibition improves inutero survival of live fetuses. Further in preterm labor induced by inflammatory response to LPS injection, upregulation of Notch-related inflammation and downregulation of angiogenesis factors was observed [76]. In both infection and inflammatory preterm labor models, we were able to rescue the phenotype by treating with $\gamma$-secretase inhibitors (GSI) [77]. This paves a way for important future direction especially since GSI is an efficient inhibitor of Notch Signaling and is currently in clinical trials for several cancers. With this, the V-ATPase and Notch crosstalk emerges to be important during normal development and indiseases like Alzheimers and various cancers [78].

\section{Wnt signaling}

The Wnt signaling pathway plays a major role in cell and tissue maintenance, polarity and differentiation. In humans, dysregulation of Wnt signaling has been implicated in cancer [79]. A classic example of dysregulated Wnt signaling is colorectal cancer wherein the loss of Adenomatous Polyposis Coli (APC), a negative regulator of Wnt signaling triggers tumorigenesis [80]. During signaling, Wnt ligands act on target cells by binding to Frizzed, Fz and LRP (low density-lipoprotein,) a cell surface receptor complex leading to disassembly of Glycogen Synthase Kinas (GSK-3) and subsequent release $\beta$-catenin. $\beta$-catenin is the main downstream mediator of Wnt pathway, which activated Wnt target oncogenes genes like c myc and cyclinD1 [81]. The (P) RR, Pro Renin Receptor also called ATP6ap2 acts as an adaptor molecule between V-ATPase and Wnt receptor complex LRP 5/6 [82]. In Xenopus and Drosophila, it has been shown that V-ATPase interacts with LRP 5/6 receptor complex and both genetic knockdown and pharmacological inhibition of V-ATPase interfere with signal transduction and significantly reduce cellular response to Wnt signaling [83, 84]. Furthermore, V-ATPase indirectly regulates Wnt signaling mediator $\beta$-catenin and Notch mediator NICD has been demonstrated through autophagy [85].

\section{TGF- $\beta$ signaling}

Mutations in the a2V gene cause Autosomal recessive Cutis Laxa (ACL) syndrome where patients present with decreased amount of extra cellular matrix proteins like Collagen resulting in wrinkly skin phenotype [86]. Supporting theses findings, a mechanistic investigation of the mutations responsible for cutis laxa in humans identified $a 2^{\mathrm{P} 405 \mathrm{~L}}$ mutation to be unstable and defective in golgi trafficking compared to wild type [87]. Further, reports point to a glycosylation defect in ACL resulting in elevated promotes transforming growth factor-beta (TGF- $\beta$ ) signaling in these patients with $a 2 \mathrm{~V}$ mutations [88]. V-ATPase promotes TGF- $\beta$ induced epithelial-mesenchymal transition of rat proximal tubular epithelial cells [89]. In addition to its effect on Notch Signaling, a2V inhibition activated Wnt pathway in TNBC and TGF- $\beta$ pathway in mammary epithelial cells [75]. This suggests that the role of $a 2 \mathrm{~V}$ in modulating signaling mediators is not exclusive to Notch. Further, these mice also displayed a reduction of total collagen due to impaired glycosylation [90].

\section{mTOR signaling}

In mTOR signaling, the Serine threonine kinase mTOR and other components of the mTOR complex 1 (mTORC1) sense amino acid availability cellular stress, and modulate growth [91] .Upon amino acid stimulation, V-ATPase activates Guanine Exchange Factor (GEF) activity of Ragulator towards RagA which in turn promotes RagC GTP hydrolysis [92]. The GTP-bound RagA and GDP-loaded RagC together recruit mTORC1 to the lysosomal surface [93]. Activated mTORC1 responds to growth factor signaling controls the regulatory switch from cell death to proliferation [94]. A recent report suggested the involvement of osteoclast proton pump regulator Atp6v1c1 in enhancing breast cancer growth by activating the mTORC1 pathway and bone metastasis by increasing V-ATPase activity [95].

\section{Conclusions}

Most studies until now have focused on the endolysosomal component of V-ATPase acidification and associated activation/degradation of signaling mediators. The signaling pathways identified to be associated with V-ATPase namely Notch, Wnt and TGF- $\beta$ surprisingly share similar expression patterns and cellular functions during both development and 


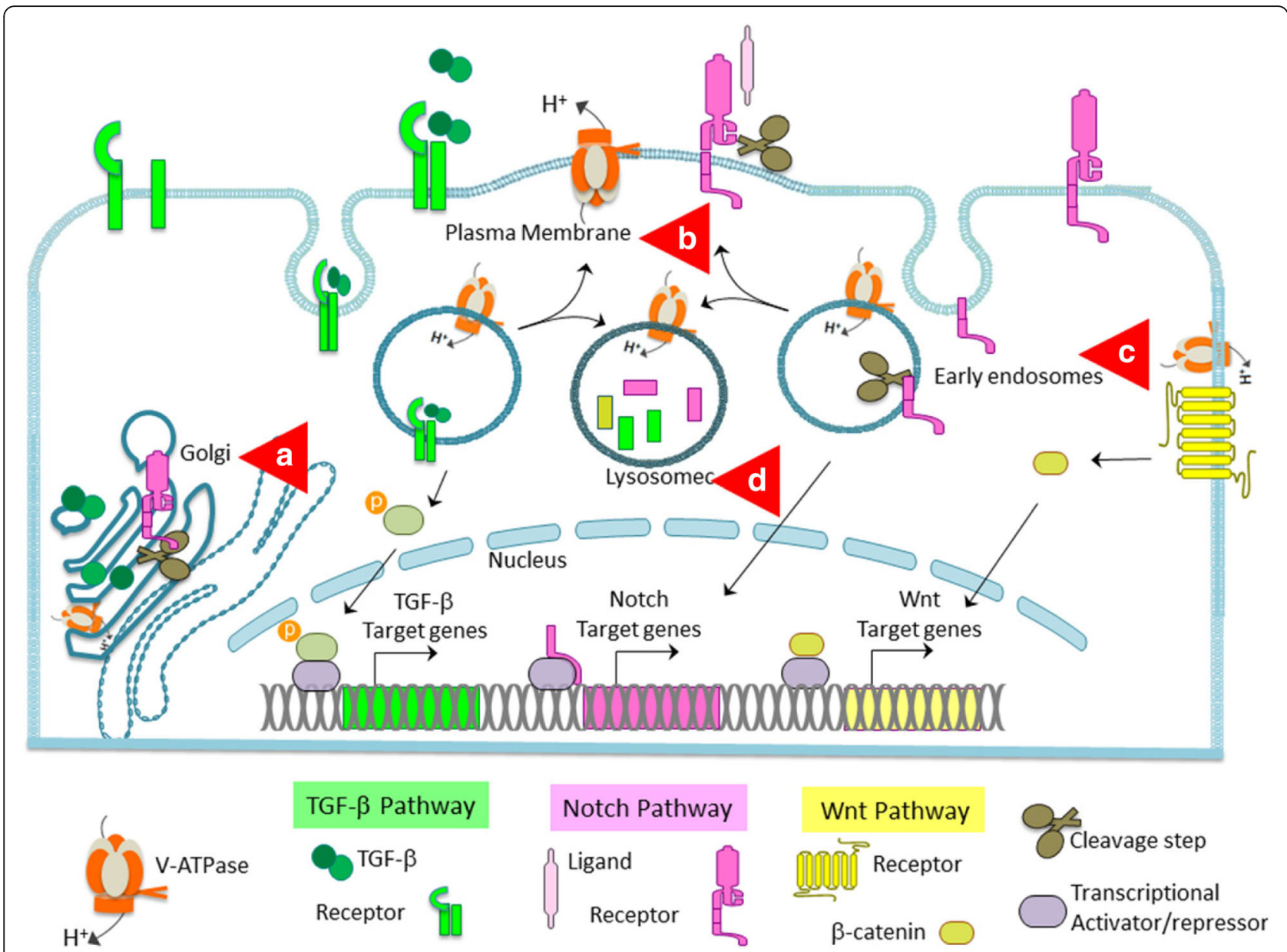

Fig. 2 The mechanistic basis of V-ATPase dependent signaling. V-ATPase (orange) acidifies intracellular vesicles thereby regulating Notch signaling and other pathways like Wnt and TGF- $\beta$, which depend on endolysosomal system for sustenance. In Notch Signaling, the Notch receptor (dark pink) is cleaved in Golgi and translocated to the plasma membrane where further cleavage of the receptor occurs in response to Notch ligand (light pink) binding. Cleaved Notch intracellular domain is translocated to nucleus activating Notch target genes. TGF- $\beta$ (dark green) protein is glycosylated in the Golgi to form mature TGF- $\beta$ and secreted into the extracellular space. TGF- $\beta$ bound to its receptor (TGF- $\beta$ R) (bright green) results in endocytosis and phosphorylation of Smad2 (olive green), which in turn activates TGF- $\beta$ target genes. During canonical Wnt signaling, the binding of ligands to the Wnt

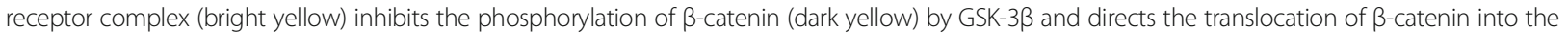
nucleus where it activates the transcription of target genes Cyclin D1 and oncogene c-Myc. V-ATPase-mediated acidification can affect signaling in the following ways: a Maturation of signaling molecules Notch receptor and TGF- $\beta$ by furin glycosylation in the golgi vesicles. $\mathbf{b}$ Cleavage and activation of pathway mediators by acid-dependent enzyme like matrix metallo proteinases (MMPs) and $\gamma$-secretase. c Maintenance of basal signaling by recycling endocytosis of both ligand and receptor. $\mathbf{d}$ Degradation of signaling molecules in lysosomes

disease. However, there are other steps of the signaling pathways known to be $\mathrm{pH}$ dependent, which warrant future investigation. Maturation of Notch and TGF- $\beta$ by glycosylation in Trans Golgi Network (TGN) activates their signaling pathways. Further $a 1$ and $a 2$ subunits of V-ATPase are important for the protein glycosylation that is a key role of the TGM. [8]. We and others have shown that surface expression of V-ATPase modulates MMPs thereby leading the proliferation ofcancer cells [43, 48]. However, V-ATPase dependent activity of ADAM/TACE has not been explored and might hold important clues for V-ATPase and signaling crosstalk mechanism. Furthermore, enzymes like $\gamma$-secretase that activate signaling pathway mediators are efficient at acidic $\mathrm{pH}$ [44]. Similarly, the involvement of V-ATPase in activation of acid proteases during lysosomal degradation to regulate signal turnover cannot be ignored [96] (See summary Fig. 2). V-ATPase could have profound effects on cell fate by influencing signaling molecules that depend on $\mathrm{pH}$. The research on V-ATPase regulation of signaling pathways is a field waiting to be explored that will have a tremendous impact in physiology and pathology.

\section{Authors' contributions}

SP and KDB conceived the original idea. SP took the lead in writing the manuscript. KDB was in charge of overall direction and planning. AK and GKK contributed to writing the manuscript and provided critical feedback. All authors read and approved the final manuscript. 


\section{Competing interests}

The authors declare that they have no competing interest.

\section{Publisher's Note}

Springer Nature remains neutral with regard to jurisdictional claims in published maps and institutional affiliations.

\section{Author details}

'Department of Microbiology and Immunology, Rosalind Franklin University of Medicine and Science, 3333 Green Bay Road, North Chicago, IL 60064, USA. ${ }^{2}$ Robert H. Lurie Comprehensive Cancer Center, Northwestern University, Chicago, IL 60611, USA

\section{Received: 22 September 2017 Accepted: 7 February 2018} Published online: 15 February 2018

\section{References}

1. Forgac M. Vacuolar ATPases: rotary proton pumps in physiology and pathophysiology. Nat Rev Mol Cell Biol. 2007;8(11):917-29.

2. Cotter K, Stransky L, McGuire C, Forgac M. Recent insights into the structure, regulation, and function of the V-ATPases. Trends Biochem Sci. 2015;40(10):611-22.

3. Marshansky V, Rubinstein JL, Gruber G. Eukaryotic V-ATPase: novel structural findings and functional insights. Biochim Biophys Acta. 2014;1837(6):857-79.

4. Stransky L, Cotter K, Forgac M. The function of V-ATPases in cancer. Physiol Rev. 2016:96(3):1071-91.

5. Nishi T, Forgac M. The vacuolar (H+)-ATPases_nature's most versatile proton pumps. Nat Rev Mol Cell Biol. 2002;3(2):94-103.

6. Wilkens S, Zhang Z, Zheng Y. A structural model of the vacuolar ATPase from transmission electron microscopy. Micron. 2005;36(2):109-26.

7. Shao E, Forgac M. Involvement of the nonhomologous region of subunit a of the yeast V-ATPase in coupling and in vivo dissociation. J Biol Chem. 2004:279(47):48663-70

8. Marshansky V, Futai M. The V-type H+-ATPase in vesicular trafficking targeting, regulation and function. Curr Opin Cell Biol. 2008;20(4):415-26.

9. Holliday LS. Vacuolar H+-ATPase: an essential multitasking enzyme in physiology and pathophysiology. New Journal of Science. 2014;2014:21.

10. Scott CC, Gruenberg J. Ion flux and the function of endosomes and lysosomes: $\mathrm{pH}$ is just the start: the flux of ions across endosomal membranes influences endosome function not only through regulation of the luminal pH. BioEssays. 2011;33(2):103-10.

11. Maxfield FR, McGraw TE. Endocytic recycling. Nat Rev Mol Cell Biol. 2004; 5(2):121-32.

12. Saftig P, Klumperman J. Lysosome biogenesis and lysosomal membrane proteins: trafficking meets function. Nat Rev Mol Cell Biol. 2009;10(9):623-35

13. Poea-Guyon S, Ammar MR, Erard M, Amar M, Moreau AW, Fossier P, Gleize V, Vitale N, Morel N. The V-ATPase membrane domain is a sensor of granular $\mathrm{pH}$ that controls the exocytotic machinery. J Cell Biol. 2013;203(2):283-98.

14. Kornak U, Reynders E, Dimopoulou A, van Reeuwijk J, Fischer B, Rajab A, Budde B, Nurnberg P, Foulquier F, Lefeber D, et al. Impaired glycosylation and cutis laxa caused by mutations in the vesicular $\mathrm{H}+$-ATPase subunit ATP6V0A2. Nat Genet. 2008;40(1):32-4.

15. Williamson WR, Hiesinger PR. On the role of v-ATPase V0a1-dependent degradation in Alzheimer disease. Commun Integr Biol. 2010;3(6):604-7.

16. O'Callaghan KM, Ayllon V, O'Keeffe J, Wang Y, Cox OT, Loughran G, Forgac M, O'Connor R. Heme-binding protein HRG-1 is induced by insulin-like growth factor I and associates with the vacuolar H+-ATPase to contro endosomal pH and receptor trafficking. J Biol Chem. 2010;285(1):381-91.

17. Strasser B, Iwaszkiewicz J, Michielin O, Mayer A. The V-ATPase proteolipid cylinder promotes the lipid-mixing stage of SNARE-dependent fusion of yeast vacuoles. EMBO J. 2011;30(20):4126-41.

18. Breton S, Brown D. New insights into the regulation of V-ATPase-dependent proton secretion. Am J Physiol Renal Physiol. 2007;292(1):F1-10.

19. Brown D, Paunescu TG, Breton S, Marshansky V. Regulation of the V-ATPase in kidney epithelial cells: dual role in acid-base homeostasis and vesicle trafficking. J Exp Biol. 2009:212(Pt 11):1762-72.

20. Karet FE. Physiological and metabolic implications of V-ATPase isoforms in the kidney. J Bioenerg Biomembr. 2005:37(6):425-9.

21. Pastor-Soler N, Pietrement C, Breton S. Role of acid/base transporters in the male reproductive tract and potential consequences of their malfunction. Physiology (Bethesda). 2005;20:417-28.
22. Jaiswal MK, Agrawal V, Katara GK, Pamarthy S, Kulshrestha A, Chaouat G, Gilman-Sachs A, Beaman KD. Male fertility and apoptosis in normal spermatogenesis are regulated by vacuolar-ATPase isoform a2. J Reprod Immunol. 2015;112:38-45.

23. Qin A, Cheng TS, Pavlos NJ, Lin Z, Dai KR, Zheng MH. V-ATPases in osteoclasts: structure, function and potential inhibitors of bone resorption. Int J Biochem Cell Biol. 2012;44(9):1422-35.

24. Paunescu TG, Jones AC, Tyszkowski R, Brown D. V-ATPase expression in the mouse olfactory epithelium. Am J Physiol Cell Physiol. 2008;295(4):C923-30.

25. Norgett EE, Golder ZJ, Lorente-Canovas B, Ingham N, Steel KP, Karet Frankl FE. Atp6v0a4 knockout mouse is a model of distal renal tubular acidosis with hearing loss, with additional extrarenal phenotype. Proc Natl Acad Sci U S A. 2012;109(34):13775-80.

26. Shine L, Kilty C, Gross J, Kennedy B. Vacuolar ATPases and their role in vision. Adv Exp Med Biol. 2014:801:97-103.

27. Breton S, Brown D. Regulation of luminal acidification by the V-ATPase. Physiology. 2013;28(5):318-29.

28. Kartner N, Manolson MF. Novel techniques in the development of osteoporosis drug therapy: the osteoclast ruffled-border vacuolar $\mathrm{H}(+$ )-ATPase as an emerging target. Expert Opin Drug Discov. 2014;9(5):505-22.

29. Smith AN, Skaug J, Choate KA, Nayir A, Bakkaloglu A, Ozen S, Hulton SA Sanjad SA, Al-Sabban EA, Lifton RP, et al. Mutations in ATP6N1B, encoding a new kidney vacuolar proton pump 116-kD subunit, cause recessive distal renal tubular acidosis with preserved hearing. Nat Genet. 2000;26(1):71-5.

30. Sennoune SR, Martinez-Zaguilan R. Plasmalemmal vacuolar H+-ATPases in angiogenesis, diabetes and cancer. J Bioenerg Biomembr. 2007;39(5-6):427-33.

31. Sennoune SR, Bakunts K, Martinez GM, Chua-Tuan JL, Kebir Y, Attaya MN, Martinez-Zaguilan R. Vacuolar H+-ATPase in human breast cancer cells with distinct metastatic potential: distribution and functional activity. Am J Physiol Cell Physiol. 2004:286(6):C1443-52.

32. Jaiswal MK, Mallers TM, Larsen B, Kwak-Kim J, Chaouat G, Gilman-Sachs A, Beaman KD. V-ATPase upregulation during early pregnancy: a possible link to establishment of an inflammatory response during preimplantation period of pregnancy. Reproduction. 2012;143(5):713-25.

33. Kwong C, Gilman-Sachs A, Beaman K. Tumor-associated a2 vacuolar ATPase acts as a key mediator of cancer-related inflammation by inducing protumorigenic properties in monocytes. J Immunol. 2011;186(3):1781-9.

34. Katara GK, Kulshrestha A, Jaiswal MK, Pamarthy S, Gilman-Sachs A, Beaman KD. Inhibition of vacuolar ATPase subunit in tumor cells delays tumor growth by decreasing the essential macrophage population in the tumor microenvironment Oncogene. 2016;35(8):1058-65. https:/doi.org/10.1038/onc.2015.159.

35. Gilman-Sachs A, Tikoo A, Akman-Anderson L, Jaiswal M, Ntrivalas E, Beaman K. Expression and role of a2 vacuolar-ATPase (a2V) in trafficking of human neutrophil granules and exocytosis. J Leukoc Biol. 2015;6:1121-31.

36. Ibrahim SA, Kulshrestha A, Katara GK, Amin MA, Beaman KD. Cancer derived peptide of vacuolar ATPase 'a2' isoform promotes neutrophil migration by autocrine secretion of IL-8. Sci Rep. 2016:6:36865.

37. Liberti MV, Locasale JW. The Warburg effect: how does it benefit cancer cells? Trends Biochem Sci. 2016:41(3):211-8

38. Torigoe $T$, Izumi $H$, Ise T, Murakami T, Uramoto H, Ishiguchi $H$, Yoshida $Y$, Tanabe M, Nomoto M, Kohno K. Vacuolar H(+)-ATPase: functional mechanisms and potential as a target for cancer chemotherapy. AntiCancer Drugs. 2002:13(3):237-43.

39. Alfarouk KO, Verduzco D, Rauch C, Muddathir AK, Adil HH, Elhassan GO, Ibrahim ME, David Polo Orozco J, Cardone RA, Reshkin SJ, et al. Glycolysis, tumor metabolism, cancer growth and dissemination. A new pH-based etiopathogenic perspective and therapeutic approach to an old cancer question. Oncoscience. 2014;1(12):777-802.

40. Gillies RJ, Robey I, Gatenby RA. Causes and consequences of increased glucose metabolism of cancers. J Nucl Med. 2008:49(Suppl 2):24S-42S.

41. Kubisch R, Frohlich T, Arnold GJ, Schreiner L, von Schwarzenberg K, Roidl A, Vollmar AM, Wagner E. V-ATPase inhibition by archazolid leads to lysosomal dysfunction resulting in impaired cathepsin B activation in vivo. Int J Cancer. 2014:134(10):2478-88.

42. Kubota S, Seyama Y. Overexpression of vacuolar ATPase $16-k D a$ subunit in 10T1/2 fibroblasts enhances invasion with concomitant induction of matrix metalloproteinase-2. Biochem Biophys Res Commun. 2000;278(2):390-4.

43. Fan SH, Wang YY, Lu J, Zheng YL, Wu DM, Zhang ZF, Shan Q, Hu B, Li MQ, Cheng W. CERS2 suppresses tumor cell invasion and is associated with decreased V-ATPase and MMP-2/MMP-9 activities in breast cancer. J Cell Biochem. 2015;116(4):502-13. 
44. Pasternak SH, Bagshaw RD, Guiral M, Zhang S, Ackerley CA, Pak BJ, Callahan JW, Mahuran DJ. Presenilin-1, nicastrin, amyloid precursor protein, and gamma-secretase activity are co-localized in the lysosomal membrane. J Biol Chem. 2003;278(29):26687-94.

45. Wojtkowiak JW, Verduzco D, Schramm KJ, Gillies RJ. Drug resistance and cellular adaptation to tumor acidic pH microenvironment. Mol Pharm. 2011; 8(6):2032-8

46. Murakami T, Shibuya I, Ise T, Chen ZS, Akiyama S, Nakagawa M, Izumi H, Nakamura T, Matsuo K, Yamada Y, et al. Elevated expression of vacuolar proton pump genes and cellular PH in cisplatin resistance. Int J Cancer. 2001;93(6):869-74.

47. Lu Q, Lu S, Huang L, Wang T, Wan Y, Zhou CX, Zhang C, Zhang Z, Li X. The expression of $\mathrm{V}$-ATPase is associated with drug resistance and pathology of non-small-cell lung cancer. Diagn Pathol. 2013;8:145.

48. Kulshrestha A, Katara GK, Ibrahim S, Pamarthy S, Jaiswal MK, Sachs AG, Beaman KD. Vacuolar ATPase 'a2' isoform exhibits distinct cell surface accumulation and modulates matrix metalloproteinase activity in ovarian cancer. Oncotarget. 2015;6(6):3797-810.

49. Wiedmann RM, von Schwarzenberg $K$, Palamidessi $A$, Schreiner $L$, Kubisch $R$, Liebl J, Schempp C, Trauner D, Vereb G, Zahler S, et al. The V-ATPaseinhibitor archazolid abrogates tumor metastasis via inhibition of endocytic activation of the rho-GTPase Rac1. Cancer Res. 2012;72(22):5976-87.

50. Huss M, Wieczorek H. Inhibitors of V-ATPases: old and new players. J Exp Biol. 2009;212(Pt 3):341-6.

51. Altan N, Chen Y, Schindler M, Simon SM. Defective acidification in human breast tumor cells and implications for chemotherapy. J Exp Med. 1998; 187(10):1583-98.

52. Kim KH, Lee MS. Autophagy-a key player in cellular and body metabolism. Nat Rev Endocrinol. 2014;10(6):322-37.

53. Gewirtz DA. The four faces of autophagy: implications for cancer therapy. Cancer Res. 2014;74(3):647-51.

54. Kissing S, Hermsen C, Repnik U, Nesset CK, von Bargen K, Griffiths G, Ichihara A, Lee BS, Schwake M, De Brabander J, et al. Vacuolar ATPase in phagosome-lysosome fusion. J Biol Chem. 2015;290(22):14166-80.

55. Mijaljica D, Prescott M, Devenish RJ. V-ATPase engagement in autophagic processes. Autophagy. 2011;7(6):666-8.

56. Carr G, Williams DE, Diaz-Marrero AR, Patrick BO, Bottriell H, Balgi AD, Donohue E, Roberge M, Andersen RJ. Bafilomycins produced in culture by Streptomyces spp. isolated from marine habitats are potent inhibitors of autophagy. J Nat Prod. 2010;73(3):422-7.

57. Mauvezin C, Nagy P, Juhasz G, Neufeld TP. Autophagosome-lysosome fusion is independent of V-ATPase-mediated acidification. Nat Commun. 2015;6:7007.

58. Sorkin A, von Zastrow M. Endocytosis and signalling: intertwining molecular networks. Nat Rev Mol Cell Biol. 2009;10(9):609-22.

59. Yoshimori T, Yamamoto A, Moriyama Y, Futai M, Tashiro Y. Bafilomycin A1, a specific inhibitor of vacuolar-type $\mathrm{H}(+)$-ATPase, inhibits acidification and protein degradation in lysosomes of cultured cells. J Biol Chem. 1991;266(26):17707-12.

60. Sun-Wada GH, Wada Y. Role of vacuolar-type proton ATPase in signal transduction. Biochim Biophys Acta. 2015;1847(10):1166-72.

61. Windler SL, Bilder D. Endocytic internalization routes required for delta/ notch signaling. Current biology : CB. 2010;20(6):538-43.

62. Le Borgne R. Regulation of notch signalling by endocytosis and endosomal sorting. Curr Opin Cell Biol. 2006;18(2):213-22.

63. Baron M. Endocytic routes to notch activation. Semin Cell Dev Biol. 2012; 23(4):437-42.

64. Vaccari T, Bilder D. The drosophila tumor suppressor vps 25 prevents nonautonomous overproliferation by regulating notch trafficking. Dev Cell. 2005;9(5):687-98.

65. Tognon E, Wollscheid N, Cortese K, Tacchetti C, Vaccari T. ESCRT-0 is not required for ectopic notch activation and tumor suppression in drosophila. PLoS One. 2014:9(4):e93987.

66. Barth JM, Hafen E, Kohler K. The lack of autophagy triggers precocious activation of notch signaling during drosophila oogenesis. BMC Dev Biol. 2012;12:35.

67. Sethi N, Yan Y, Quek D, Schupbach T, Kang Y. Rabconnectin-3 is a functional regulator of mammalian notch signaling. J Biol Chem. 2010;285(45):34757-64

68. Sorensen EB, Conner SD. gamma-secretase-dependent cleavage initiates notch signaling from the plasma membrane. Traffic. 2010;11(9):1234-45.

69. Lange $C$, Prenninger S, Knuckles $P$, Taylor V, Levin M, Calegari F. The H(+) vacuolar ATPase maintains neural stem cells in the developing mouse cortex. Stem Cells Dev. 2011;20(5):843-50.

70. Valapala M, Hose S, Gongora C, Dong L, Wawrousek EF, Samuel Zigler J Jr, Sinha D. Impaired endolysosomal function disrupts notch signalling in optic nerve astrocytes. Nat Commun. 2013;4:1629.
71. Wada Y, Sun-Wada GH. Positive and negative regulation of developmental signaling by the endocytic pathway. Curr Opin Genet Dev. 2013;23(4):391-8.

72. Barth JM, Kohler K. How to take autophagy and endocytosis up a notch. Biomed Res Int. 2014;2014:960803.

73. Lee JH, Yu WH, Kumar A, Lee S, Mohan PS, Peterhoff CM, Wolfe DM, Martinez-Vicente M, Massey AC, Sovak G, et al. Lysosomal proteolysis and autophagy require presenilin 1 and are disrupted by Alzheimer-related PS1 mutations. Cell. 2010;141(7):1146-58.

74. Pamarthy S, Jaiswal MK, Kulshreshtha A, Katara GK, Gilman-Sachs A, Beaman KD. The vacuolar ATPase a2-subunit regulates notch signaling in triplenegative breast cancer cells. Oncotarget. 2015;6(33):34206-20.

75. Pamarthy S, Mao L, Katara GK, Fleetwood S, Kulshreshta A, Gilman-Sachs A, Beaman KD. The V-ATPase a2 isoform controls mammary gland development through notch and TGF-beta signaling. Cell Death Dis. 2016;7(11):e2443.

76. Jaiswal MK, Agrawal V, Pamarthy S, Katara GK, Kulshrestha A, Gilman-Sachs A, Beaman KD, Hirsch E. Notch signaling in inflammation-induced preterm labor. Sci Rep. 2015;5:15221.

77. Agrawal V, Jaiswal MK, Pamarthy S, Katara GK, Kulshrestha A, Gilman-Sachs A, Hirsch E, Beaman KD. Role of notch signaling during lipopolysaccharideinduced preterm labor. J Leukoc Biol. 2016;100(2):261-74. https://doi.org/10. 1189/jlb.3HI0515-200RR.

78. Lee JH, McBrayer MK, Wolfe DM, Haslett LJ, Kumar A, Sato Y, Lie PP, Mohan $P$, Coffey EE, Kompella U, et al. Presenilin 1 maintains lysosomal $\mathrm{ca}(2+)$ homeostasis via TRPML1 by regulating VATPase-mediated lysosome acidification. Cell Rep. 2015;12(9):1430-44.

79. Polakis P. Wnt signaling in cancer. Cold Spring Harb Perspect Biol. 2012;4(5).

80. Sebio A, Kahn M, Lenz HJ. The potential of targeting Wnt/beta-catenin in colon cancer. Expert Opin Ther Targets. 2014;18(6):611-5.

81. Baarsma HA, Konigshoff M, Gosens R. The WNT signaling pathway from ligand secretion to gene transcription: molecular mechanisms and pharmacological targets. Pharmacol Ther. 2013;138(1):66-83.

82. Ichihara A. (Pro)renin receptor and vacuolar H(+)-ATPase. Keio J Med. 2012; 61(3):73-8.

83. Cruciat CM, Ohkawara B, Acebron SP, Karaulanov E, Reinhard C, Ingelfinger $D$, Boutros $M$, Niehrs $C$. Requirement of prorenin receptor and vacuolar H+-ATPase-mediated acidification for Wnt signaling. Science. 2010:327(5964):459-63.

84. Rousselle A, Sihn G, Rotteveel M, Bader M. (Pro)renin receptor and V-ATPase: from drosophila to humans. Clin Sci (Lond). 2014;126(8):529-36.

85. Gao C, Cao W, Bao L, Zuo W, Xie G, Cai T, Fu W, Zhang J, Wu W, Zhang X, et al. Autophagy negatively regulates Wnt signalling by promoting Dishevelled degradation. Nat Cell Biol. 2010;12(8):781-90.

86. Guillard M, Dimopoulou A, Fischer B, Morava E, Lefeber DJ, Kornak U, Wevers RA. Vacuolar H+-ATPase meets glycosylation in patients with cutis laxa. Biochim Biophys Acta. 2009;1792(9):903-14.

87. Esmail S, Kartner N, Yao Y, Kim JW, Reithmeier RAF, Manolson MF. Molecular mechanisms of cutis laxa and distal renal tubular acidosiscausing mutations in V-ATPase a subunits, ATP6V0A2 and ATP6V0A4. J Biol Chem. 2018;

88. Fischer B, Dimopoulou A, Egerer J, Gardeitchik T, Kidd A, Jost D, Kayserili H, Alanay Y, Tantcheva-Poor I, Mangold E, et al. Further characterization of ATP6VOA2-related autosomal recessive cutis laxa. Hum Genet. 2012;131(11):1761-73.

89. Cao X, Yang Q, Qin J, Zhao S, Li X, Fan J, Chen W, Zhou Y, Mao H, Yu X. VATPase promotes transforming growth factor-beta-induced epithelialmesenchymal transition of rat proximal tubular epithelial cells. Am J Physiol Renal Physiol. 2012;302(9):F1121-32.

90. Katara GK, Kulshrestha A, Mao L, Wang X, Sahoo M, Ibrahim S, Pamarthy S, Suzue K, Shekhawat GS, Gilman-Sachs A, et al. Mammary epithelium-specific inactivation of V-ATPase reduces stiffness of extracellular matrix and enhances metastasis of breast cancer. Mol Oncol. 2018;12(2):208-23.

91. Moschetta M, Reale A, Marasco C, Vacca A, Carratu MR. Therapeutic targeting of the mTOR-signalling pathway in cancer: benefits and limitations. Br J Pharmacol. 2014;171(16):3801-13.

92. Zoncu R, Bar-Peled L, Efeyan A, Wang S, Sancak Y, Sabatini DM. mTORC senses lysosomal amino acids through an inside-out mechanism that requires the vacuolar H(+)-ATPase. Science. 2011;334(6056):678-83.

93. Kim YC, Guan KL. mTOR: a pharmacologic target for autophagy regulation. J Clin Invest. 2015;125(1):25-32.

94. Bar-Peled L, Sabatini DM. Regulation of mTORC1 by amino acids. Trends Cell Biol. 2014;24(7):400-6. 
95. McConnell M, Feng S, Chen W, Zhu G, Shen D, Ponnazhagan S, Deng L, Li YP. Osteoclast proton pump regulator Atp6v1c1 enhances breast cancer growth by activating the MTORC1 pathway and bone metastasis by increasing V-ATPase activity. Oncotarget. 2017:8(29):47675-90.

96. Kissing S, Saftig P, Haas A. Vacuolar ATPase in phago(lyso)some biology. Int J Med Microbiol. 2017.

Submit your next manuscript to BioMed Central and we will help you at every step:

- We accept pre-submission inquiries

- Our selector tool helps you to find the most relevant journal

- We provide round the clock customer support

- Convenient online submission

- Thorough peer review

- Inclusion in PubMed and all major indexing services

- Maximum visibility for your research

Submit your manuscript at www.biomedcentral.com/submit 